SHS Web of Conferences 2, 00012 (2012)

DOI: $10.1051 /$ shsconf $/ 20120200012$

(C) Owned by the authors, published by EDP Sciences, 2012

\title{
Influence of accessibility of services on Quality of Life of school children with Cerebral Palsy in Latvia
}

\author{
A. Greitane ${ }^{1}$, I. Ceiciniece ${ }^{1}$, L. Cibule ${ }^{2}$ and U. Teibe ${ }^{2}$ \\ ${ }^{1}$ Rehabilitation Center "Mes esam lidzas", Latvia \\ ${ }^{2}$ Rĭga Stradinš̌ University, Latvia
}

\begin{abstract}
The aim of the study was to investigate the influence of accessibility of services and health related factors on Quality of Life (QoL) of school children with Cerebral Palsy (CP) in Latvia. 80 children 47 boys and 33 girls with $\mathrm{CP}$ and their parents from all regions of Latvia took part in the study. Mean age of participants was $11,7(\mathrm{SD} \pm 3,5)$ years. 50 children without diagnosed chronic diseases formed the control group. Validated evaluation tools were used in the study (KIDSCREEN-52, GMFCS- E\&R). The results show lower QoL in children with CP in comparison with their healthy peers and European children. QoL is influenced by the level of gross motor functions, lack of coordination of services, lack of rehabilitation infrastructure and presence of environmental barriers in Latvia.
\end{abstract}

Key words: cerebral palsy, quality of life, rehabilitation services

Cerebral Palsy (CP) is the most common physical disability in childhood. It describes a group of permanent disorders of the development of movements and posture, causing activity limitations that are attributed to non progressive disturbances that occurred in the developing fetal and infant brain. The motor disorders of $\mathrm{CP}$ are often accompanied by disturbances of sensation, perception, cognition, communication, and behavior, by epilepsy, and by secondary musculosceletal problems (Rosenbaum et al., 2006).

The impact of $\mathrm{CP}$ on individuals varies widely, from someone who cannot mobilize independently, has little communication and has associated impairments such as epilepsy and severe intellectual impairment, to someone who has very mild impairment of motor control (Bax, Gillberg, 2010).

Overall, the CP prevalence in Europe is between 2 and 3 per 1000 live births. This rate increases to 40-100 per 1000 live births among babies born very early or with very low birth weight (Paneth, 2006).

Unfortunately there are no data about the prevalence of CP cases in Latvia. Nevertheless we see more and more children with $\mathrm{CP}$ at schools, in the kindergartens and in our neighbourhood.

After the acceptance of the law of Disability in Latvia in 2010 several questions have raised - "How do we protect our children and young people with disabilities?", "What do we know about them?", "How do we establish a framework that seeks to support them?"

Assessing QoL in children with disabilities is a good way to learn more about them. QoL is defined by WHO as "an individual's perception of their position in life in the context of the culture and value systems in which they live, and in relation to their goals, expectations, standards and concerns" (SCPE, 2007) QoL is therefore subjective and so must be reported by the individual concerned whenever possible. This is a challenge when assessing children's QoL because they have typically been regarded as unreliable respondents. Evidence is accumulating that children can self-report QoL reliably if their emotional development, cognitive ability, and reading level are taken into account (Colver, SPARCLE group, 2006).

This is an Open Access article distributed under the terms of the Creative Commons Attribution License 2.0, which permits unrestricted use, distribution, and reproduction in any medium, provided the original work is properly cited. 


\section{SHS Web of Conferences}

The common challenge faced by researchers in the field of $\mathrm{CP}$ is inability of many children to communicate themselves thus, the need to rely on the information from the caregiver or parents (Lim, Wong, 2009).

The disproportion between the accessibility of services due to the current socioeconomic situation and the legislation in Latvia was the reason to study the real needs of the children with $\mathrm{CP}$ and their families.

Our goals were: to analyze QoL of children with CP, to compare QoL between children with CP and their healthy peers, to assess the level of agreement concerning QoL between children with CP and their parents, to analyze QoL of children with $\mathrm{CP}$ in relation with accessibility of rehabilitation and social services.

\section{Materials and methods}

This was a prospective, descriptive study of children with CP of Latvia. The total number of participants in this study was 210 . It represents 80 children with $\mathrm{CP}-47(58,8 \%)$ boys and $33(41,3 \%)$ girls, age $8-18$, mean age of participants was $11,7(\mathrm{SD} \pm 3,5)$ years. 80 parents of those children also took part in the study. 50 children without diagnosed chronic diseases formed the control group.

The data used in this investigation were collected in all regions of Latvia by the multidisciplinary team of the Rehabilitation Center "Mes esam lidzas".

Clinical presentations of CP were described as in SCPE Reference and Training Manual (Paneth, 2006).

This classification is made on the basis of the predominant neurological findings as spastic $\mathrm{CP}$-bilateral spastic, unilateral spastic, dyskinetic $\mathrm{CP}$-dystonic, choreo-athetotic and ataxic $\mathrm{CP}$. Classification tree for $\mathrm{CP}$ subtypes, $\mathrm{CP}$ inclusion and exclusion criteria were taken into account.

As an indicator of gross motor function level the Gross Motor Function Classification System Expanded and Revised (GMFCS- E\&R) (Palisano et al., 2007) was used. It classifies a person's ability to self initiate movements related to sitting and walking. Typical performances rather than maximal performance were rated using a five level scale. The GMFCS- E\&R has evidence of reliability and validity.

We used Bimanual Fine Motor Function (Beckung, Hagberg, 2002) - an assessment which takes into account symmetrical or asymmetrical involvement of the upper limbs for hand function evaluation.

Self-reported Health related Quality of Life (HRQoL) was assessed with KIDSCREEN-52 (RavensSieberer et al., 2005) a modern instrument with excellent psychometric properties, which uses questions derived from focus-group work with children across Europe to assess the QoL of healthy children and young people. The KIDSCREEN-52 instrument contains the following dimensions- physical well-being, psychological well-being, moods and emotions, self perseption, autonomy, parent relation and home life, peers and social support, school environment, bullying and financial resources.

For children with CP it was used in the Study of Participation of Children with Cerebral Palsy Living in Europe (SPARCLE) (Colver, SPARCLE group, 2006).

A Survey for Accessibility of Services (SAS) was used to describe the current situation of accessibility of services in families with CP children.

Child/family questionnaire was developed by the research team to obtain information on the child and family. Demographic questions were indicators of child's age, sex and domestic situation. Ethics approval was received from the State Medical Ethics committee.

We trained the research associates to use the above mentioned evaluation instruments. Children and families were therefore visited by researchers competent both to administer questionnaires to parents, caregivers and to engage children for completion of their questionnaires. The visits took place in the child's education facility and lasted about 90 minutes. Children with communication difficulties in addition to their $\mathrm{CP}$ were included by ensuring assistance from a parent or teacher. Children with 


\section{Int. Conf. SOCIETY. HEALTH. WELFARE; Congr. of Rehabilitation Doctors of Latvia}

learning difficulties were not able to report their QoL, in this case the reports of parents or caregivers were used.

We used software SPSS 18,0 for the statistical analysis.

\section{Results}

43 school children with CP could self report on their OoL, in 7 cases when the child was intellectually handicaped only the parents' questionnaire was filled.

Before the statistical data analysis Cronbach's alpha test was used to measure the internal consistency of data. Its value for the $\mathrm{CP}$ group was 0,877 , for the parents $-0,892$ and the control group $-0,920$. These values indicate good consistency of 52 questionaire items.

All types of CP were represented in our study, the distribution of subtypes were close to European data from SCPE study (Table 1).

Table 1 describes also the severity of impairment of gross motor function (GMFCS E\&R). The $5^{\text {th }}$ level of GMFCS E\&R (children need total assistance, independent movements are not possible) was represented only by 3 school children $(3.8 \%)$.

Figure 1 reflects the overall QoL research results for children with CP. They show lower scores in all dimensions when compared with children from the European QoL study and in 9 scores when compared with healthy peers from Latvia.

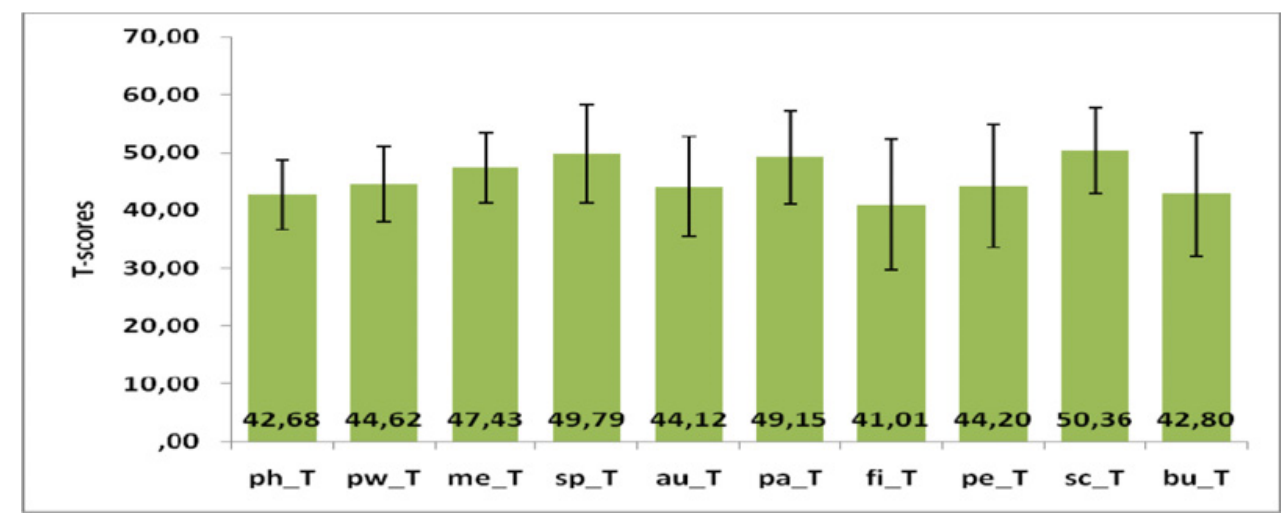

Figure 1. $\mathrm{T}$ - scores of $\mathrm{CP}$ children for the dimensions - ph_T - physical well-being, pw_T - psychological wellbeing, me_T - moods and emotions, sp_T - self perseption, au_T - autonomy, pa_T - parent relation and home life, fi_T - financial resources, pe_T - peers and social support, sc_T - school environment, bu_T - bullying.

We found reliability of statistical data between the dimensions of physical well-being and psychological well-being (Spearman's rank correlation coefficient $r=0,54$ ), physical well-being and peers, social support $r=-0,58$, psychological well-being and moods, emotions $r=-0,50$, psychological well-being and autonomy $\mathrm{r}=0,46$, psychological well-being and parent relation, home life $r=0,48$, parent relations, home life and peers and social support $r=0,45$.

Studying the gross and fine motor function levels statistical reliability was found between GMFCS level and physical well-being (Pearson correlation coefficient $r=-0,51 ; p=0$ ), GMFCS level and peers, social support $(\mathrm{r}=0,39 ; \mathrm{p}=0,005)$.

Children reported higher QoL than their parents. Results show that level of agreement differ between children and parents in the dimensions of physical well-being $(t=4.644)$, psychological well-being $(t=3,418)$, social support/participation $(t=1,904)$.

Evaluating additional factors as place of residence (Riga and regions of Latvia) and family life (full or single parent family) we found statistical reliability between the place of residence and the dimension of financial resources $(r=-0,370 ; p=0,008)$, place of residence and the dimension 
SHS Web of Conferences

Table 1. Included CP children, impairment and socio-economic characteristics.

\begin{tabular}{|c|c|c|}
\hline Classification & Category & No. $(\%)$ of children \\
\hline \multicolumn{3}{|l|}{ Impairment } \\
\hline \multicolumn{3}{|c|}{ Cerebral palsy type } \\
\hline & Unilateral spastic & $15(18,7)$ \\
\hline & Bilateral spastic & $47(58,8)$ \\
\hline & Dyskinetic & $10(12,5)$ \\
\hline & Ataxic & $8(10,0)$ \\
\hline \multicolumn{3}{|c|}{ Gross Motor Function Classification System } \\
\hline & GMFCS 1 & $31(38,8)$ \\
\hline & GMFCS 2 & $11(13,6)$ \\
\hline & GMFCS 3 & $15(18,8)$ \\
\hline & GMFCS 4 & $20(25,0)$ \\
\hline & GMFCS 5 & $3(3,8)$ \\
\hline \multicolumn{3}{|c|}{ Bimanual Fine Motor Function } \\
\hline & BFMF 1 & $28(35,0)$ \\
\hline & BFMF 2 & $20(25,0)$ \\
\hline & BFMF 3 & $12(15,0)$ \\
\hline & BFMF 4 & $16(20,0)$ \\
\hline & BFMF 5 & $4(5,0)$ \\
\hline \multicolumn{3}{|c|}{ Associated impairments } \\
\hline & Vision & $49(61,3)$ \\
\hline & Hearing & $12(15,0)$ \\
\hline & Seizures & $25(31,3)$ \\
\hline \multicolumn{3}{|c|}{ Communication } \\
\hline & Normal or difficulty, but uses speech & $73(91,5)$ \\
\hline & No formal communication & $7(8,5)$ \\
\hline \multicolumn{3}{|l|}{ Schooling } \\
\hline & Mainstream & $60(75,0)$ \\
\hline & Special school & \\
\hline & level A & $5(6,2)$ \\
\hline & level B & $9(11,3)$ \\
\hline & level C & $6(7,5)$ \\
\hline \multicolumn{3}{|c|}{$\begin{array}{l}\text { Socio-economic characteristics: } \\
\text { Area of living }\end{array}$} \\
\hline & Riga & $50(62,5)$ \\
\hline & Region & $30(37,5)$ \\
\hline \multicolumn{3}{|l|}{ Family status } \\
\hline & Full family & $53(66,2)$ \\
\hline & Single parent family & $27(33,8)$ \\
\hline
\end{tabular}

of peers and social support $(r=0,310 ; p=0,03)$. These relationships reflect higher QoL in the dimension of financial resources for children who live in Riga. Children from Riga had higher scores in the dimensions of psychological well-being and school environment, however it is not statistically relieable $-\mathrm{p}>0,05$.

Children who live in families with both parents reflect higher QoL in all dimensions except in the dimension of moods and emotions and social acceptance. Statistical reliability can be seen between the family situation and dimension of psychological well-being $(r=-0,410 ; \mathrm{p}=0,003)$. 
Int. Conf. SOCIETY. HEALTH. WELFARE; Congr. of Rehabilitation Doctors of Latvia

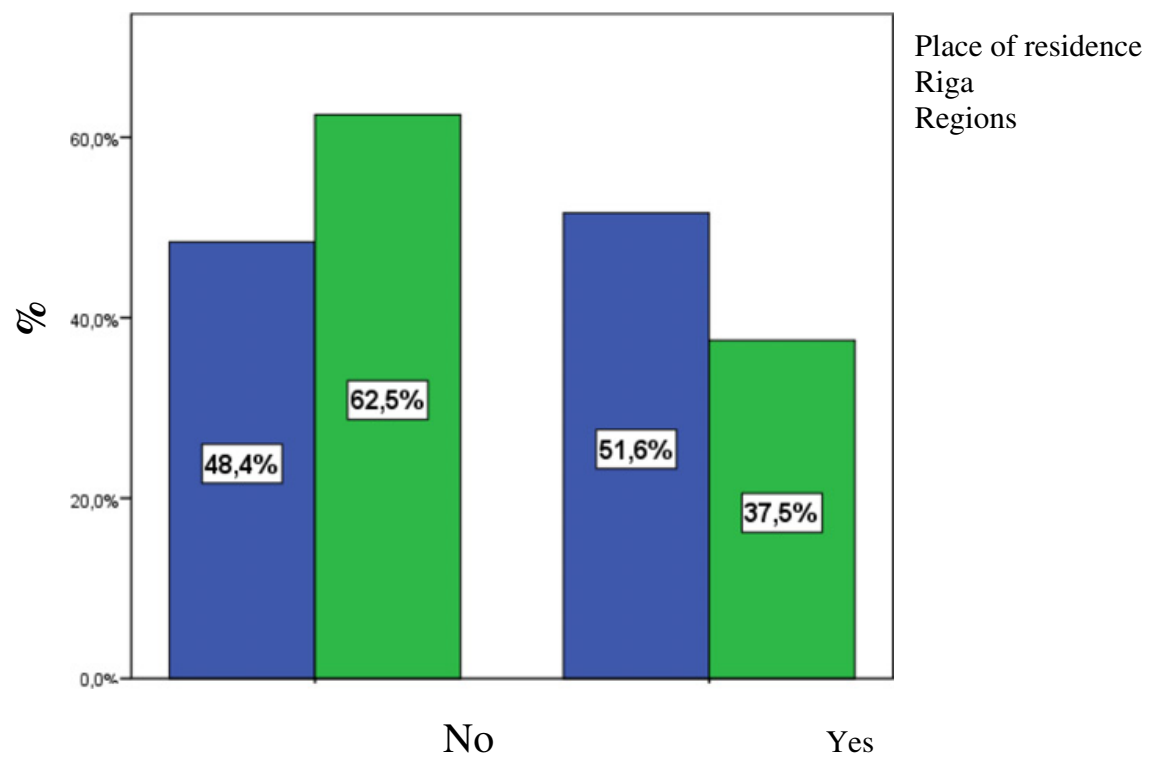

Figure 2. Availability of rehabilitation services in places of residence.

56 parents ( $70 \%$ out of 80 persons involved in the research) responded to the part of the questionnaire about rehabilitation services and their availability. In the majority of cases parents acknowledged that their children receive appropriate $(17.9 \%)$ or partially appropriate $(62.5 \%)$ rehabilitation services. $14.3 \%$ of parents thought that their children receive improper, but $5.3 \%$-do not receive rehabilitation at all.

More than a half $(62.5 \%)$ of respondents living in regions noted that rehabilitation services are not available in their community. However, it must be added that the situation in Riga is just a little better (Figure 2).

Parents named as most efficient and necessary services the following: physiotherapy -42 , ride therapy - 21 times, massage and swimming - 29 times, occupational therapy -18 , speech therapy -15 and psychologist's help in 9 cases.

\section{Discussion}

Children with CP often have impairments of learning, hearing, vision, communication and epilepsy in addition to their motor ones and so are representatives of the wider population of disabled children (Colver, SPARCLE group, 2006).

Our results show that abnormal vision had $61.3 \%$, hearing impairment $-15.0 \%$, epilepsy $-31.3 \%$, intellectual disability and learning disorders $-25.1 \%$. Only $22.5 \%$ of children with CP did not have any associated pathology, $48.8 \%$ had one, $27.6 \%$ - two, but $1.3 \%$ even three. Our study showed lower scores in the dimensions of physical well-being and finances for the CP children with associated pathologies.

When planning clinical services, this syndromic approach is valuable as the differing syndromes will require different interventions to manage the condition, although curing the primary cause is not possible (Colver, SPARCLE group, 2006).

Canadian CP research group has found that participation of children with $\mathrm{CP}$ differs based on gross motor function. Children with higher physical ability had fewer activity limitations and participation restrictions than those with lower physical ability (Palisano et al., 2011).

Participation directly correlates with QoL. Our study reflects the influence of gross motor function level (GMFCS E\&R) on dimensions of physical weel-being - Pearson correlation coefficient $\mathrm{r}=-0,51 ; \mathrm{p}=0$, as well as gross motor function and social acceptance $\mathrm{r}=0,39 ; \mathrm{p}=0,005$. 


\section{SHS Web of Conferences}

Which means - children with higher functional level reported higher physical weel-being and social acceptance.

The same tendencies reflect another Canadian study which stresses that among children with CP higher intelligence and gross motor function were associated with increased participation and QoL (Majnemer et al., 2008).

Family members are the most important people in child's life (King et al., 2006). Supportive family environments and financial resources influence participation by providing children with opportunities and experiences (Law et al., 2006). The family structure and relationships, family activity orientation and the socio-demographics and family income are proposed to have a direct effect on participation and QoL and indirect effect through services.

Children of this study who live in families with both parents reflect higher QoL in all dimensions except in the dimension of moods and emotions and social acceptance. Availability, accessibility, coordination and communication, and responsiveness to family information needs are tenets of familycentered services hypothesized to optimize children's activity and participation (Mihaylov et al., 2004).

Parents in our study defined difficulties in accessibility of services - lack of coordination, delays in reception and unavailability of therapies, also barriers of home and school environment (only 5,4\% of participants reported about fit of children with CP and home environment), lack of finances for services of personal assistants. That influences autonomy of children with CP $(\mathrm{m}=41.05)$.

Parents also think that family orientated services are highly unavailable (28 out of 56). Although the situation has improved in comparison with 2005, when $90 \%$ parents considered that services are not family orientated. Most often parents stress the need of the following services - respite care, personal assistance for child and residences of independent living for the young people.

Higher gross motor function, higher enjoyment, more effective adaptive behavior, younger age and higher family activity orientation are associated with higher intensity of participation and QoL of children (Palisano et al., 2011).

Accessibility of parks, playgrounds and public buildings, availability of community programs, wheelchair accessible transportation, and activity accommodations are environmental factors that might influence participation and QoL of children with CP.

Healthcare professionals have an important role in supporting social and community participation of children with $\mathrm{CP}$, including enhancing family knowledge of community resources and advocating for inclusive environments (Palisano et al., 2011). Our research clearly shows the need to include the CP children and their parents in the decision-making about the rehabilitation goals and strategies, as well as the most useful therapies for the individual.

Children with CP are a group with relatively stable impairment where participation and QoL will be influenced by social and educational environmental factors as well as by medical interventions (Orlin et al., 2010).

\section{Conclusions}

Children with CP in Latvia have lower QoL in comparison with their healthy peers and with their peers in Europe, but at the same time they report higher QoL than their parents. QoL of children with CP is influenced by their functional abilities - mainly gross motor function as well as by the associated pathologies.

QoL is influenced by social and educational environmental factors as well as by medical interventions which facilitate gross motor functions.

Infrastructure for providing rehabilitation services in Latvia is still under development and in the majority of cases children with $\mathrm{CP}$ at present do not receive proper rehabilitation services. Community based services are available only for a half of the children involved in the research and still in the majority of cases parents use in patient rehabilitation services for their children. 
Int. Conf. SOCIETY. HEALTH. WELFARE; Congr. of Rehabilitation Doctors of Latvia

Larger populations of disabled children in Latvia need to be studied quantitatively to determine how and why quality of life varies between children with comparable severity of impairment.

Monitoring the rate of the condition in the population and thus providing data for planning and organization of health, social and educational services for young people with Cerebral Palsy is of vital importance.

The study was initiated and funded by the Latvia Children's Fund.

\section{References}

[1] Bax, M., Gillberg, C. (2010) Comorbidities in developmental disorders. Mac Keith Press, London, p. 20-39.

[2] Colver, A. and the SPARCLE group. (2006) Study protocol: SPARCLE - a multi-centre European study of the relationship of environment to participation and quality of life in children with cerebral palsy. BMC Public Health, 2006, 6, p.105.

[3] Dodd, K., Imms, C., Taylor, N. (2010) Physiotherapy and occupational therapy for people with cerebral palsy. Mac Keith Press, London, p. 1-20.

[4] King, G., Law, M., Hanna, S. et al. Predictors of the leisure and recreation participation of children with physical disabilities a structural equation modeling analysis. Child health care, 2006, 35, p. 209-34.

[5] Law, M., King, G., King, S. et al. Patterns of participation in recreation and leisure activities among children with complex physical disabilities, Dev.Med.Child Neurology, 2006, 48, p. 337-42.

[6] Lim, M.S.Y., Wong, C.P., (2009) Impact of cerebral palsy on the quality of life in patients and their families. Neurology Asia, 2009, 14, p. 27-33.

[7] Majnemer, A., Shevell, M., Law, M. et al. Participation and enjoyment of leisure activities in school aged children with cerebral palsy. Dev.Med.Child Neurology, 2008, 50, p. 751-8.

[8] Mihaylov, S. et al. Identification and description of environmental factors that influence participation of children with cerebral palsy. Dev.Med.Child Neurology, 2004, 46, p. 299-304; 299.

[9] Orlin, M.N., Palisano, R.J., Chiarello, L.A. et al. Participation in home, extracurricular, and community activities among children and young people with cerebral palsy. Dev.Med.Child Neurology, 2010, 52, p. 160-6.

[10] Palisano, R., Rosenbaum, P., Bartlett, D., Livingston, M. (2007) GMFCS-E\&R.

[11] avens-Sieberer et al. and the European KIDSCREEN group. (2005) KIDSCREEN-52 quality-oflife measure for children and adolescents. Expert Review of Pharmacoeconomics \& Outcomes Research, 5 (3), p. 353-364.

[12] Palisano, R.J. et al. Determinants of intensity of participation in leisure and recreational activities by children with cerebral palsy. Dev.Med. Child. Neurology, 2011, 53, p. 142-149.

[13] Rosenbaum, P. (2003) Cerebral palsy; what parents and doctors want to know. BMJ, 326, p. 970-974.

[14] Rosenbaum et al. (2006) The definition and classification of cerebral palsy. Dev.Med.Child Neurology, 49 (Suppl. 109), p. 8-14.

[15] SCPE (2007) Reference and Training Manual - R\&TM.

[16] WHOQOL Assessment Group: Development and general psychometric properties. Soc.Sci.Med., 1998, 46, p. 1569-85. 